REVISTA INFOCIENCIA

Vol. 12 Núm. 1 / 2018

\title{
Comparación de Métodos de Búsqueda Robótica para apoyar al Desminado Humanitario
}

\section{Comparison of Robotic Search Methods to support Humanitarian Demining}

\author{
Marcelo Saavedra Alcoba, German Pereira Muñoz. \\ Carrera de ingeniería Mecatrónica, Escuela Militar de Ingeniería \\ La Paz, Bolivia
}

Correspondiente de autores: msaavedraa@adm.emi.edu.bo, gpereiram@doc.emi.edu.bo

Recibido: junio 2018, Publicado: diciembre 2018

\begin{abstract}
Resumen - El presente trabajo propone apoyar a la búsqueda de minas explosivas mediante los robots que ya es una realidad. Se llevó a cabo un análisis exhaustivo comparando algoritmos de búsqueda en diferentes situaciones. Para este objetivo se creó un modelo de un ambiente realista simulado en la plataforma Stage. Se realizaron un total de 6000 pruebas comparando cuatro modelos de búsqueda en el simulador. Los resultados muestran que el algoritmo propuesto tiene una tasa de detección de $72,3 \%$ que aproximadamente es mayor en un $40 \%$ sobre un método simple de exploración. La principal contribución reportada en este trabajo es la propuesta de un algoritmo autónomo con una moderada tasa de detección.
\end{abstract}

Palabras Claves- Desminado humanitario, robótica humanitaria, robot buscaminas, robot inteligente.

\begin{abstract}
The present work proposes to support the search for explosive mines using robots. We carry out an exhaustive analysis comparing search algorithms in different situations. For this purpose, a realistic simulated environment model was created on the Stage platform. A total of 6000 trials were carried out comparing four search models in the simulator. The results show that the algorithm proposed by us has a detection rate of $72.3 \%$ which is approximately $40 \%$ higher than a simple method of exploration. There contribution reported in this work, is the proposal of an autonomous algorithm with a moderate detection rate.
\end{abstract}

Keywords-Humanitarian demining, humanitarian robotics, minesweeper robot, intelligent robot.

\section{INTRODUCCIÓN}

Las minas antipersonales en el campo de batalla son un procedimiento contra intrusos o enemigos, a diferencia de una bomba o un proyectil de artillería que explota cuando se acerca o hace contacto con el objetivo, una mina queda oculta hasta que la activa una persona, un animal o la proximidad de un vehículo [ CITATION Dia16 \l 22538 ]1. Es por esta razón que los esfuerzos para erradicarlos son de carácter internacional, a este proceso se le conoce como desminado y es un proceso peligroso que consume mucho tiempo. Utilizando robots para este fin, se puede disminuir el peligro, acelerar el desminado y aumentar su fiabilidad y seguridad [ CITATION Por14 \l 22538 ]2.

En el presente trabajo se propone encontrar una estrategia óptima de búsqueda de minas simulando algunos problemas encontrados, por ejemplo saber que algoritmo de búsqueda utilizar, que pasa si existen obstáculos en el ambiente, que pasa si el ambiente a recorrer no es cuadrado y tiene sectores accidentados, que pasa si el sensor de detección de minas no tiene una alta confiabilidad.

\section{ESTADO DEL ARTE}

Los avances de la búsqueda de minas antipersonales mediante robots tienen algo más de 20 años, desde robots profesionales hasta robots de bajo costo [ CITATION Non02 \l 16394 ]3.

Ismail et. al. [ CITATION Ism14 \l 22538 ]4, estudiaron una visión general de las técnicas de detección de minas utilizando robots, concluyendo que las tecnologías existentes aumentan la probabilidad de detección, reducen la tasa de falsas alarmas y planifican escenarios de implementación utilizables. También menciona que no existe un método único para la detección eficiente de minas terrestres.

Actualmente existe el campeonato mundial robótico MinesWeepers, que es un concurso donde cada equipo presenta un robot teleoperado o autónomo para encontrar minas antipersonales enterradas y encima del suelo, a su vez el robot también debe ir creando automáticamente un mapa de las minas detectadas.

En el concurso MinesWeepers la búsqueda de las minas se realiza en un área de $20 \times 20$ metros y cada mina puede estar en un cuadrado de un metro, entonces generalmente los participantes lo resuelven escaneando todo el ambiente, pero en un ambiente más real es muy probable que no se tenga un área cuadrada pequeña, tampoco sabremos si las minas tienen separaciones de un metro y el ambiente de exploración puede estar en un lugar altamente accidentado, entonces la exploración robótica necesaria se convierte en un problema de búsqueda ya conocido en el mundo de la robótica móvil.

La búsqueda de objetos en un entorno real es compleja, porque el objeto a buscar no siempre estará visible, de hecho en el trabajo [CITATION Saa15 \l 22538 ]5, se menciona que los autores Ye y Tsotsos en su trabajo titulado "Sensor Planning for 3D object search", demuestran matemáticamente que la búsqueda de un objeto arbitrario en 
un espacio 3D es un problema NP-completo [ CITATION Mal13 \ 16394 ]6. En [ CITATION Lon13 \l 16394 ]7, se realiza un método de búsqueda no informado, basado en la maximización de la probabilidad de exploración.

\section{METODOLOGÍA DE SIMULACIÓN}

Proponemos un algoritmo para mejorar el proceso de búsqueda de minas terrestres en un ambiente irregular. El diagrama del esquema general del sistema se puede ver en la Fig. 1, donde se tiene un bloque de Estrategia, un bloque de actuación, un bloque de Percepción y un bloque de Actualización de Mapas.

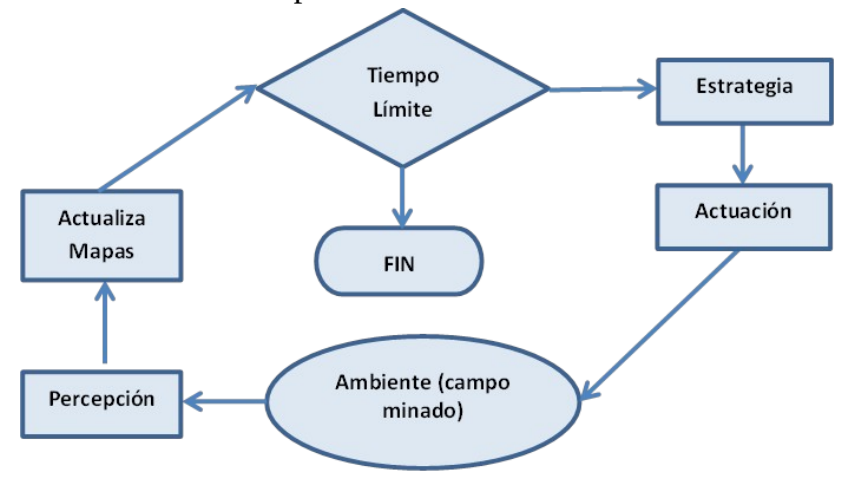

Fig. 1. Diagrama del esquema general del sistema

A continuación se explicará con detalle el simulador utilizado y los bloques que se realizaron en el simulador:

A.

Simulador robótico STAGE.

La ventaja de un simulador es que se puede usar como herramienta para la evaluación, depuración y ajuste del programa de control antes de llevar la aplicación al robot real [ CITATION Bal10 \l 21514 ]8.

El proyecto Player/Stage fue fundado por Brian Gerkey, Richard Vaughan y Andrew Howard en el año 2000, en los laboratorios de robótica de la Universidad de California del Sur (ver Fig. 2).
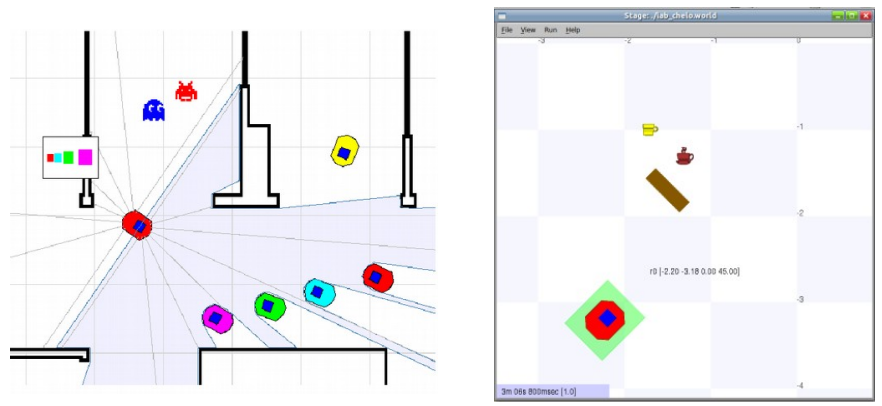

Fig. 2. Plataforma de simulación: Stage

\section{B. Bloque Percepción.}

La primera acción del robot es captar las señales de los sensores (inductivo y de proximidad) y procesarlas. Las salidas de este módulo son 3 casos posibles que son la detección de la mina, la detección de obstáculos y la no detección.
En el primer caso, si se detecta la mina se procede marcar en el mapa y continúa con el proceso de búsqueda. En el segundo caso el robot cambia de dirección, En el último caso se continúa con la búsqueda. El proceso termina cuando se llega a un tiempo límite programado.

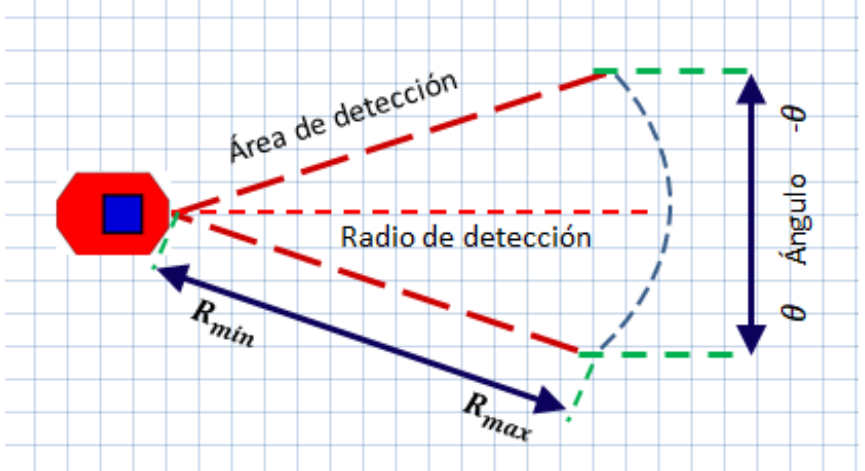

Fig. 3. Grilla de certeza de un sensor de detección en Stage

El simulador Stage trae una herramienta de detección llamado fiducial detector model, que simula una detección en $2 \mathrm{D}$, es decir en un plano horizontal.

La estructura de esta función tiene como entradas un valor de rango mínimo y rango máximo (por sus siglas en ingles range_min y range_max), que son intervalos de distancia y el ángulo fov (field of view), que es el ángulo del campo de visión del robot en grados, como se puede ver en la Fig. 3. Para reconocer a los objetos se utiliza la función range_max_id que detecta la id del objeto (cuál objeto es el detectado).

Cualquier objeto con un id conocido en el plano 2D y que este dentro del fov será detectado por el robot. Esta función es muy útil a la hora de simular una exploración robótica con detección de objetos.

\section{Caso a)}

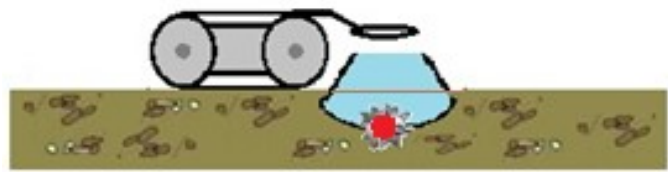

Caso b)

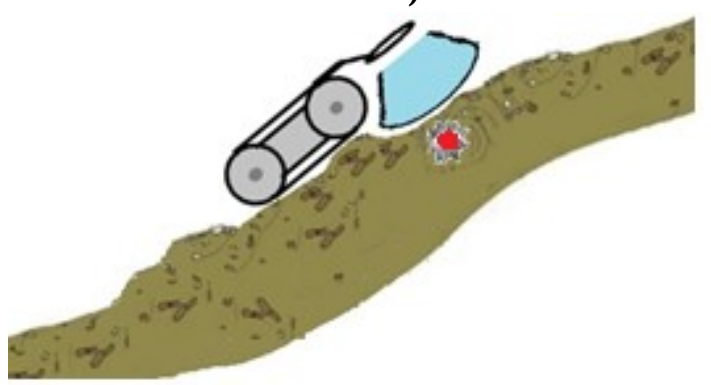

Fig. 4. Caso a ) Objeto con alto índice de detección, Caso b ) Objeto con bajo índice de detección

Con el fin de simular la búsqueda de minas, se realizó la simulación de 3 casos: 
i. Modelo del ambiente de prueba de $20 \times 20$ metros.

ii. Modelo del robot que se propone usar

iii. Modelo del sensor inductivo para la detección de minas en cada frame dependiente del terreno (regular o irregular) donde se encuentra el robot (ver Fig. 4).

Para el tercer caso, se realizó una probabilidad de detección de un $25 \%$ para las minas que se estarían en una zona accidentada y para las minas que están en una zona normal se realizó una probabilidad de detección de un 85\% por cada frame.

\section{Bloque de Actualización de Mapas.}

Se programó en el simulador una rejilla donde se traza el recorrido del robot y la posición donde se detecta la mina, vale aclarar que se da por supuesto el modelo de localización y que la navegación no se desorienta mediante el recorrido.

D. Bloque de Actuación.

Luego del proceso de estrategia de búsqueda, en este módulo se realizará una acción sobre el robot (desplazamiento) buscando las minas mencionadas. Una vez obtenido el punto y dirección donde el robot debe llegar, en este módulo se realiza la acción definida incluyendo un comportamiento reactivo usando el sensor de proximidad del robot para evitar colisionar con los obstáculos, o pisar las minas detectadas en el ambiente.

\section{ESTRATEGIAS DE BÚSQUEDA}

Se estudian cuatro métodos de búsqueda de objetos en el bloque de estrategia, que se detallan a continuación.

A.

Búsqueda mediante vehículos Braitenberg.

Los vehículos de Braitenberg [CITATION Bra84 U 16394 ]9, son experimentos robóticos basados en movimientos de animales cuando se acercan o alejan por un estímulo. En el trabajo de [ CITATION MarcadorDePosición1 ll 16394 ]10 se puede ver una comparación de estos métodos demostrando que el comportamiento "Explorador" tiene un mejor rendimiento en cuestiones de búsqueda.

\section{B. Búsqueda en Zigzag}

Una exploración en Zigzag está basada en el patrón compuesto trazando un camino entre dos líneas paralelas, que puede ser regulares o irregulares. En el trabajo de [ CITATION Oss09 \l 16394 ]11 , se presenta un sistema que es capaz de navegar rápidamente a través de un denso campo de obstáculos, recorriendo la mayor parte del mapa, por lo que se decidió usarlo en este caso en un problema de búsqueda no informada.

\section{Búsqueda Probabilística.}

En el trabajo [CITATION Lon01 V 16394 ]12, los autores realizaron una búsqueda probabilística bayesiana. Donde el término $p\left(a_{i, j}\right)$ representa la probabilidad de que un objeto (en este caso una mina) esté en la celda $(i, j)$. La probabilidad $p\left(a_{0}\right)$ Se trata como un caso especial, y representa la probabilidad de que el objeto esté fuera de la región de búsqueda. Las observaciones negativas $z_{A}$ proveen una información valiosa para el proceso de búsqueda, y pueden utilizarse para calcular una probabilidad actualizada $p\left(a_{i, j} \mid\right.$ $\left.z_{A}\right)$.

Considerando el siguiente caso:

$$
p\left(a_{i, j} \mid z_{A}=\text { false }\right)=\frac{p\left(z_{A}=\text { false } \mid a_{i, j}\right) p\left(a_{i, j}\right)}{p\left(a_{o}\right)+\sum_{i, j} p\left(z_{A}=\text { false } \mid a_{i, j}\right) p\left(a_{i, j}\right)}
$$

$$
p\left(a_{0} \mid z_{A}=\text { false }\right)=\frac{p\left(a_{0}\right)}{p\left(a_{o}\right)+\sum_{i, j} p\left(z_{A}=\text { false } \mid a_{i, j}\right) p\left(a_{i, j}\right)}
$$

Cada vez que no se detecte una mina, es decir que la observación $z_{A}=$ false, se marca la vista en forma del cono de detección reduciendo la probabilidad de encontrar minas en ese lugar. La ruta para la búsqueda de un objeto dado puede ser creado mediante la generación de puntos de vista óptimos en cada iteración, es decir, que en cada movimiento se decide la nueva pose observando diferentes nuevas poses aleatorias, si el robot ya pasó por un lugar, como se explicó antes, este será marcado como baja probabilidad de encontrar nuevas minas. Es por esa razón que estos nuevos puntos de vista óptimos aleatorios, pretenden no navegar por espacios recorridos con anterioridad, por lo que en general este método de búsqueda, explora la mayoría del mapa. El punto de vista óptimo se genera a partir de un conjunto de poses aleatorios alcanzable en un tiempo fijo, y seleccionando la que maximiza la probabilidad de encontrar el objeto en el área [ CITATION Ayd10 v 16394 ]13:

$$
\underset{k=1 . . N}{\operatorname{argmax}} \sum_{i=1}^{n} \sum_{j=1}^{n} p\left(a_{i, j}\right) V\left(a_{i, j}, k\right)
$$

Donde $\mathrm{N}$ es el número de poses candidatas, y $\mathrm{V}$ se define como:

$$
V\left(a_{i, j}, k\right)= \begin{cases}1, & \text { if } a_{i, j} \text { is inside the } \mathrm{k}^{\text {th }} \text { view cone } \\ 0 & \text { otherwise }\end{cases}
$$

Entre los modelos de búsqueda no informada, por los resultados que estudiamos, creemos que este es uno de los más eficientes.

\section{Búsqueda Hibrida Propuesta}

Según las búsquedas no informadas estudiadas en este trabajo tanto la búsqueda en Zigzag y la búsqueda probabilista son muy eficientes, por lo tanto nosotros 
proponemos un algoritmo sencillo pero eficiente para poder realizar la búsqueda de minas. Esta propuesta consiste en realizar una exploración en Zig-Zag por la mitad del tiempo límite, con esto se habrá cubierto gran parte del mapa, luego utilizar la búsqueda probabilística sabiendo que su navegación buscará explorar lugares no visitados con anterioridad. En teoría con este método se debería poder recorrer mayor parte del ambiente.

\section{RESULTADOS}

\section{A. Configuración de los experimentos}

Los experimentos se realizaron en un conjunto de 10 mapas, cada uno con cincuenta objetos (minas). Cada mapa contiene 40 objetos para detectar (minas) con alta probabilidad de detección y 10 minas que tienen un índice menor para su detección, el tamaño de los mapas es de $20 \times$ 20 metros. Para cada experimento se tomó en cuenta la incidencia del sensor inductivo (detector de metales) del robot mediante un cono de visión con apertura de 45, 70, 90, 120 y 180 grados (ver Fig. 5), es decir se realizaron pruebas con sensores de diferentes tamaños para poder probar como mejora la búsqueda cuando se tiene un detector de mayor alcance.
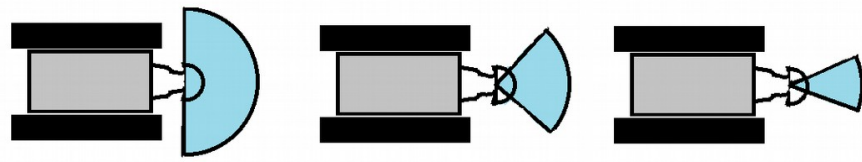

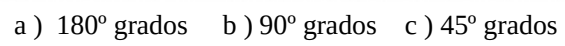

Fig. 5. Representación de sensores inductivos de mayor alcance.

Para cada mapa se distribuyeron las 50 minas en posiciones aleatorias. En la Fig. 6 se puede apreciar la forma de los mapas utilizados para realizar los experimentos. Un sensor láser de Player/Stage se utiliza para evitar colisiones.

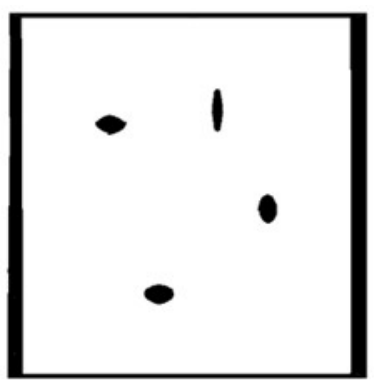

a ) Mapa 1

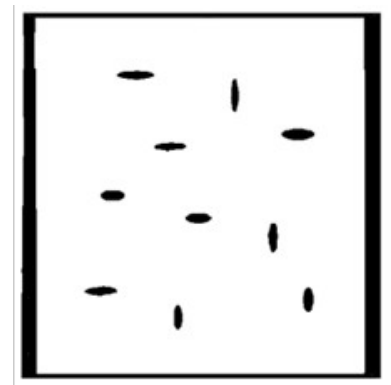

b ) Mapa 10
Fig. 6. Mapas representando obstaculos existentes en el ambiente por el robot

\section{B. Resultados Preliminares}

Cuatro algoritmos de búsqueda de objetos son comparados en el mismo conjunto de mapas. Estos algoritmos son:

\section{1) Búsqueda mediante explorador de Braitenberg.}

Los resultados obtenidos con el explorador de Braitenberg son relativamente bajos, ya que este modelo repite con bastante frecuencia los lugares ya explorados. Los resultados se pueden ver en la Fig. 7.

\section{2) Búsqueda mediante exploración Zigzag.}

Los resultados obtenidos con el método de Zigzag son relativamente buenos, ya que, debido a su forma geométrica, el espacio de búsqueda tiene un ancho mayor por donde pasa el robot, detectando así, casi todas las minas que se encuentran en su camino. Los resultados se pueden ver en la Fig. 8.
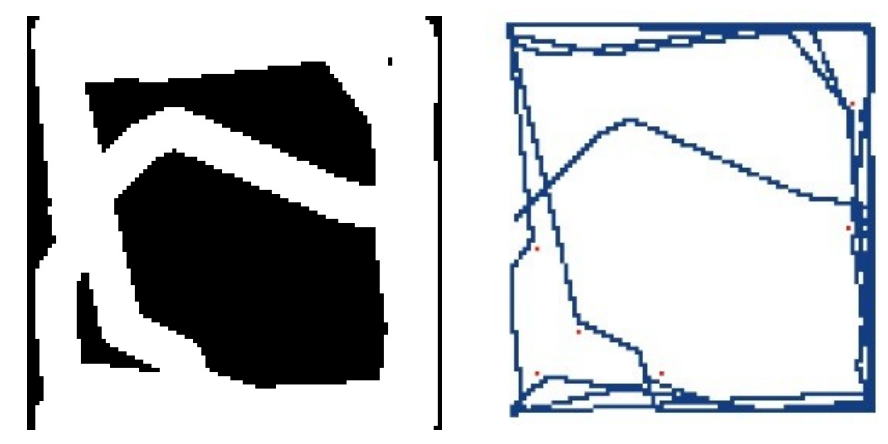

Fig. 7. Resultados gráficos del explorador de Braitenberg
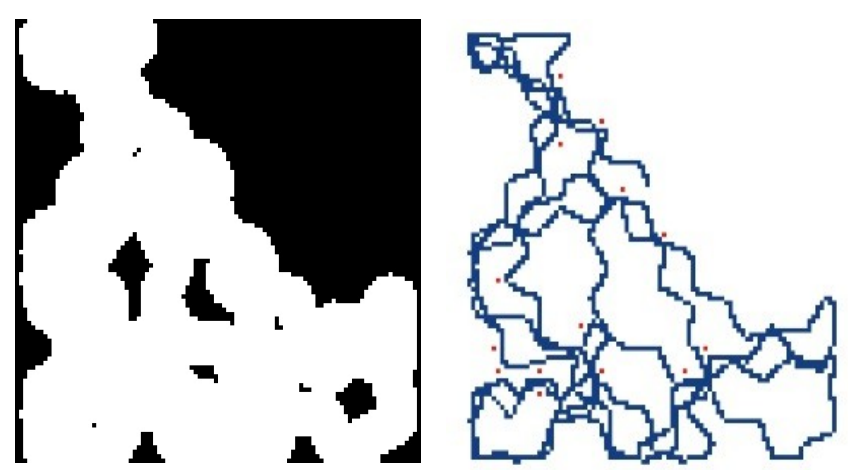

Fig. 8. Resultados obtenidos con el método Zigzag

\section{1) Búsqueda Probabilística.}

Se calcula un mapa de probabilidad $\mathrm{p}\left(\mathrm{a}_{\mathrm{i}, \mathrm{j}}\right)$ al mismo tiempo que el mapa del recorrido del robot, mientras existan detecciones negativas se va marcando el mapa, si es que se encuentra una mina se marca un circulo alrededor del objeto para que el robot no pase por ese lugar. Luego se busca nuevas minas por lugares donde exista mayor probabilidad. Los resultados se pueden ver en la Fig. 9.
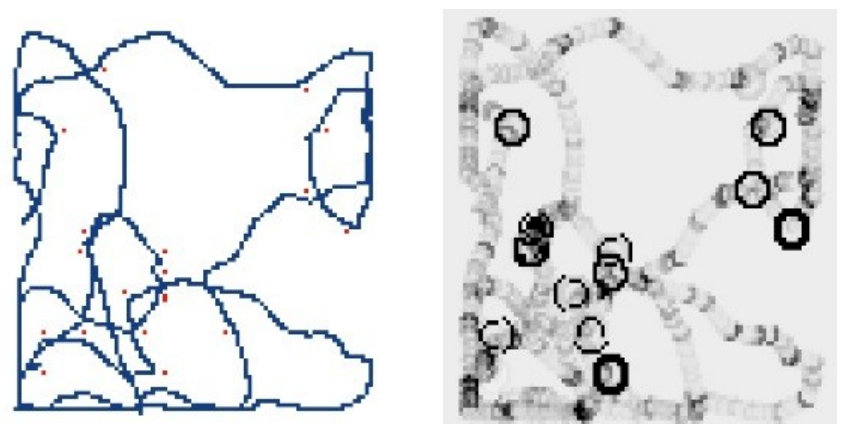

Fig. 9. Resultados obtenidos con el método probabilístico 
2) Búsqueda híbrida entre el método Zigzag y el método probabilístico.

Luego de varios experimentos, se presenta esta búsqueda hibrida que es la propuesta más óptima, donde la mitad del tiempo de exploración se realizó con el método Zigzag y la otra mitad con el método probabilístico. Los resultados se pueden ver en la Fig. 10, claramente a diferencia del método solo Zigzag que tiende a repetir demasiadas veces un mismo lugar y el probabilístico que tiende a moverse por todo el mapa pero de forma más lenta, este método combinado detecta más minas que en los anteriores casos. Entonces primero se realiza la exploración en Zigzag, y después de un cierto tiempo se realiza la búsqueda probabilística que tiende a explorar los lugares no visitados. Dada la configuración del mapa y la posición de las minas, en varias ocasiones, si o si el robot tiene que pasar por algunos lugares ya visitados con anterioridad, porque aunque tengan menor probabilidad, es el único camino posible.

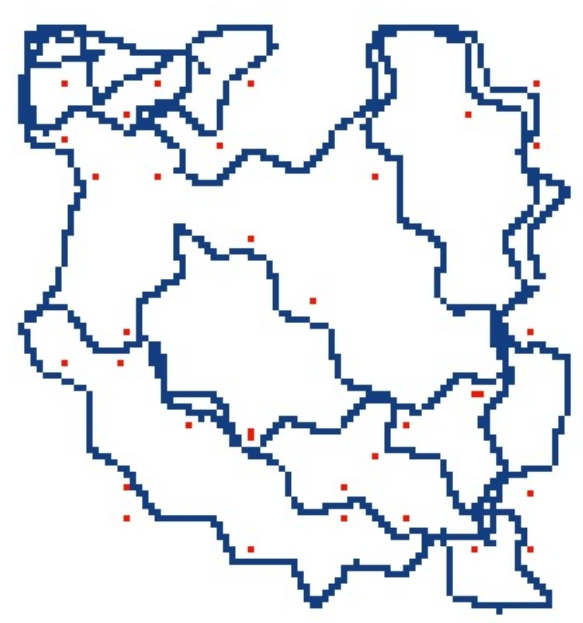

Fig. 10. Modo de exploración del método híbrido

\section{C. $\quad$ Tasa de Detección}

Los experimentos se validaron en los computadores del Laboratorio de Robótica de la Escuela Militar de Ingeniería. Se realizaron 30 ejecuciones por cada mapa (10 mapas), variando el cono de detección del robot en 45, 70, 90, 120 y 180 [grados].

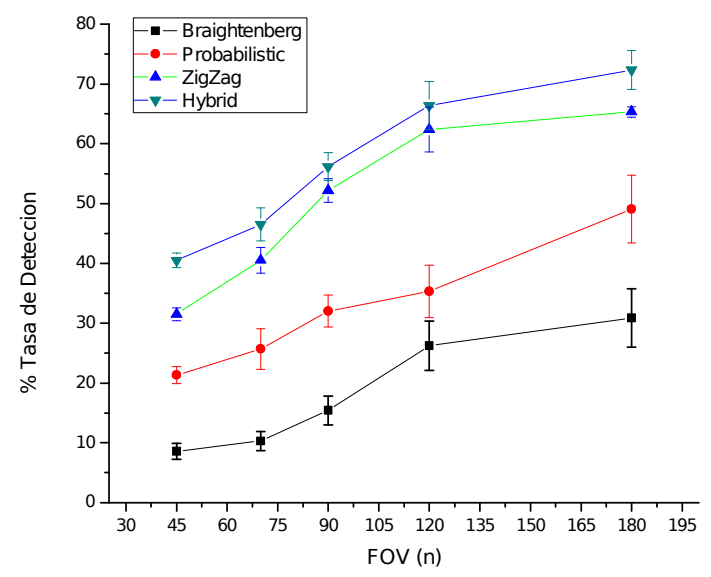

Fig. 11. Efecto de la incidencia del sensor en 5 ángulos diferentes
En la Fig. 11 se puede observar que el algoritmo de Braitenberg tiene porcentajes de detección más bajos, mientras que el modelo probabilístico y la exploración con el método de Zigzag obtienen un mejor rendimiento de detección. Por esta razón, se realiza una combinación de ambos métodos (probabilístico y Zigzag) para maximizar la detección de minas.

En total se realizaron 1500 pruebas por cada método, es decir 6000 experimentos. (ver Tabla 1).

TABLA 1. RESUltADOS FINALES

\begin{tabular}{|l|c|c|c|c|c|}
\hline \multirow{2}{*}{$\begin{array}{l}\text { PROMEDIO DE LA TASA DE } \\
\text { DETECCIÓN }\end{array}$} & \multicolumn{5}{|c|}{ ESCALAS DE VISTA DEL SENSOR } \\
\cline { 2 - 6 } & $\mathbf{4 5}^{\mathbf{0}}$ & $\mathbf{7 0}^{\mathbf{}}$ & $\mathbf{9 0}^{\mathbf{}}$ & $\mathbf{1 2 0}^{\mathbf{}}$ & $\mathbf{1 8 0}^{\mathbf{0}}$ \\
\cline { 2 - 6 }$\mu$ Búsqueda por Braightenberg & 8,6 & 10,3 & 15,4 & 26,2 & 30,9 \\
\hline$\mu$ Búsqueda Probabilística & 21,3 & 25,7 & 32,0 & 35,3 & 49,1 \\
\hline$\mu$ Búsqueda en Zigzag & 31,5 & 40,5 & 52,2 & 62,4 & 65,3 \\
\hline$\mu$ Búsqueda Hibrida propuesta & 40,5 & 42,5 & 59,2 & 66,4 & 72,3 \\
\hline
\end{tabular}

\section{CONCLUSIONES}

Se realizó y validó cuatro modelos de búsqueda en un entorno simulado de un área minada; estas pruebas se realizaron en las mismas condiciones al medir una tasa de detección (TD) que varía la incidencia de la detección del sensor.

Analizando el rendimiento de todos los experimentos y variando la incidencia de la detección del sensor, se puede observar que esto afecta considerablemente el rendimiento de todos los métodos. A partir de los resultados, es evidente que el "método híbrido" obtiene un mejor rendimiento que los otros métodos. Esto ocurre porque se usan dos métodos que maximizan el área de escaneo en un tiempo más corto.

Como trabajo a futuro se propone realizar más experimentos, como evaluar la extensión de la trayectoria de los métodos comparados, que daría una idea del tiempo de exploración de cada método y la consecuente eficiencia.

\section{RECONOCIMIENTOS}

Nuestro agradecimiento a la Carrera de Ingeniería Mecatrónica de la Escuela Militar de Ingeniería por financiar parcialmente esta investigación y a la Dirección Nacional de Investigación Ciencia Y Tecnología - DNICYT de la EMI por recibirla y tomar el tiempo de revisarla.

\section{REFERENCIAS}

[1] Diario_digital_PUBLICO. (2016, Apr.) ¿En qué países siguen matando las minas antipersona? [Online].

http://www.publico.es/internacional/paises-siguen-matando-minasantipersona.html

[2] D. Portugal , L. Marquez, and M. Armada, "Deploying field robots for humanitarian demining: Challenges, requirements and research trends," in Int. Conf. Climbing Walking Robots (CLAWAR), Poznan - Polonia, 2014.

[3] Kenzo Nonami, "Development of Autonomous Mine Detection SixLegged Walking Robot for Humanitarian Demining," The Proceedings of the Asian Conference on Multibody Dynamics, pp. 1-8, 2002. 
[4] Ahmed Ismail, Mohammed Elmogy, and Hazem ElBakry, "Landmines Detection Using Autonomous Robots: A Survey," International Journal of Emerging Trends \& Technology in Computer Science (IJETTCS), 2014.

[5] M. Saavedra Alcoba, Búsqueda de objetos mediante conocimiento semántico en ambientes interiores, Universidad de Chile, Ed.: Tesis Magister, 2015, vol. 10.

[6] Carlos Eduardo Maldonado, "Un problema fundamental en la investigación: Los problemas P vs. NP," Revista Logos Ciencia \& Tecnología, vol. 4, no. 2, pp. 10-20, 2013.

[7] J. Ruiz del Solar, P. Loncomilla, and M. Saavedra, "A Bayesian framework for informed search using convolutions between observation likelihoods and spatial relation masks," in 16th International Conference on Advanced Robotics (ICAR), Montevideo Uruguay, 2013, pp. 1-8.

[8] T. R. Balaguer, Diseño e implementación de una capa de software para el control de robots mediante Player/Stage. Madrid España: M.Sc. Thesis, Departamento de Informática, 2010.

[9] Marcelo Saavedra Alcoba, Mariela Gutierrez Callejas, and Luigi Enríquez Paz, "Análisis de Comportamientos de Vehículos de Braitenberg para la búsqueda robótica usando El Robot LEGO EV3," Fides Et Ratio [online], vol. 12, no. 12, pp. 155-166, 2016.

[10] Marcelo Saavedra Alcoba and Luigi Enríquez Paz, "Diseño de un algoritmo de búsqueda informada mediante el Simulador Robótico Stage," Fides et Ratio - Revista de Difusión cultural y científica de la Universidad La Salle en Bolivia, vol. 10, no. 10, pp. 39-59, 2015.

[11]A. Ossino and E. Barnett, "Path planning for robot-assisted rapid prototyping of ice structures," Centre for Intelligent Machines, Department of Mechanical Engineering, Montreal, Canada, Tech. Rep. TR-CIM-09-02 2009.

[12] Patricio Loncomilla, J. Ruiz-del-Solar, and Marcelo Saavedra Alcoba, "A Bayesian based Methodology for Indirect Object Search," Journal of Intelligent and Robotic Systems, vol. 90, pp. 45-63, 2018.

[13] A. Aydemir, K. Sjöö, and P. Jensfelt, "Object search on a mobile robot using relational," in Conference on Intelligent Autonomous Systems (IAS-11), Sweden, 2010. 